\title{
Treatment of chronic mandibular dislocations by eminectomy: Follow-up of 10 cases and literature review
}

\author{
Belmiro-Cavalcanti do Egito Vasconcelos ${ }^{1}$, Gabriela-Granja Porto ${ }^{2}$, José-Pacheco-Martins-Ribeiro Neto ${ }^{3}$, \\ César-Freire de Melo Vasconcelos ${ }^{4}$
}

${ }^{1}$ DDS PhD, Senior Lecturer in Oral and Maxillofacial Surgery. Director of the Master and PhD programs in Oral and Maxillofacial Surgery, University of Pernambuco (Recife-Brazil)

${ }^{2}$ DDS MSc, Postgraduate Student of the PhD program in Oral and Maxillofacial Surgery, University of Pernambuco. RecifeBrazil

${ }^{3}$ MD, Assistant Professor of Medicine - FBV/IMIP, Recife-Brazil

${ }^{4}$ Graduate Student of Medicine - FBV/IMIP, Recife-Brazil

Correspondence:

Faculdade de Odontologia de Pernambuco

Departamento de Cirurgia e Traumatologia BMF

Av. General Newton Cavalcanti, 1650

Camaragibe - PE

54753-220 Brazil

belmiro@pesquisador.cnpq.br

Received: $11 / 11 / 2008$

Accepted: 20/05/2009

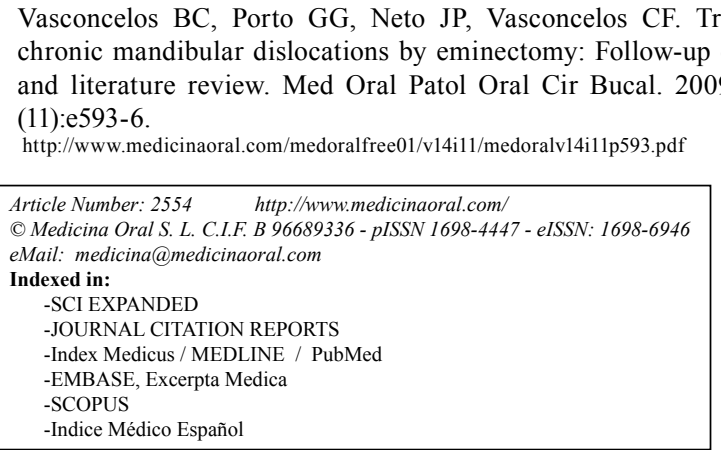

\begin{abstract}
Introduction: Temporomandibular joint (TMJ) dislocation is defined as an excessive forward movement of the condyle beyond the articular eminence, with complete separation of the articular surfaces and fixation in that position.

Objectives: To report ten cases treated by eminectomy for chronic mandibular dislocations, to evaluate the results of these surgeries and make a critical review of the literature.

Methods: The sample was obtained from the records of the Department of Oral and Maxillofacial Surgery, University of Pernambuco and comprises cases submitted to chronic mandibular dislocation treatment by eminectomy between 2002 and 2007. Pre- and postoperative assessment included a thorough history and physical examination to determine the maximal mouth opening, presence of pain and sounds, frequency of luxations, recurrence rate and presence of facial nerve paralysis.

Results: The mean maximal mouth opening in the preoperative period was $48.4 \pm 8.5 \mathrm{~mm}$ and in the postoperative period it was $41.3 \pm 5.0 \mathrm{~mm}$. No facial nerve paralysis or recurrence was observed.

Conclusion: The treatment of chronic mandibular dislocations by eminectomy was shown to be efficient in relationship to the postoperative maximal mouth opening, recurrence and articular function.
\end{abstract}

Key words: Temporomandibular joint disorders, surgery, joint instability. 


\section{Introduction}

Temporomandibular joint (TMJ) dislocation is defined as an excessive forward movement of the condyle beyond the articular eminence with complete separation of the articular surfaces and fixation in that position $(1,2)$. It is commonly associated with poor development of the articular fossa, laxity of the temporomandibular ligament or joint capsule and excessive activity of the lateral pterygoid and infrahyoid muscles due to drug use or disease $(1,2)$.

A variety of therapeutic approaches designed to limit the forward excursion of the condylar head have been applied, such as intra-capsular injection of sclerosing solutions (3), lateral pterygoid myotomy (4), scarification of the temporalis tendon (5) and bone grafting augmentation or application of a well-designed alloplastic impediment with vitallium mesh or titanium plates (6). Another type of treatment is to reduce the eminence, thereby permitting free movement of the condyle $(6,7)$. In this paper, 10 cases of chronic mandibular dislocation treated by eminectomy are reported. A descriptive statistical analysis is also presented, as well as a critical review of the literature.

\section{Patients and Methods}

A retrospective evaluation of 10 consecutively treated patients with chronic mandibular dislocations was conducted at the Department of Oral and Maxillofacial Surgery, University of Pernambuco, Recife-PE, Brazil, between 2002 and 2007. The patients were treated by eminectomy.
The following criteria were used for inclusion in the study: patients with episodes of at least five dislocations per month; inability to perform jaw movements to smile, speak or eat; and failure of conservative treatment methods, such as orientation to self-limit jaw movements and the use of a chin cup.

Radiographic examination included panoramic radiographs and conventional tomography to determine the height of the articular eminence. Preoperative assessment included a thorough history and physical examination to determine the maximal mouth opening (MMO), age, sex, frequency and recurrence of the dislocations (1). The patients were pre- and postoperatively examined and classified according to the House and Brackmann (8) grading system in order to detect any signs of facial nerve paralysis or paresthesia. The period of recovery from any paralysis was also observed.

- Surgical procedures:

Exposure of the TMJ was done by the preauricular approach described under general anesthesia. After exposure and identification of the articular eminence, it was removed using a \# 703 drill. The jaw movements were then checked for interference and any required adjustments made (Figure 1).

\section{Results}

The data of the patients treated by eminectomy according to age, sex, MMO, presence of pre- and postoperative pain and sounds, follow-up and frequency of luxations are shown in Table 1.

The mean age of the patients in this study was 31.4 years

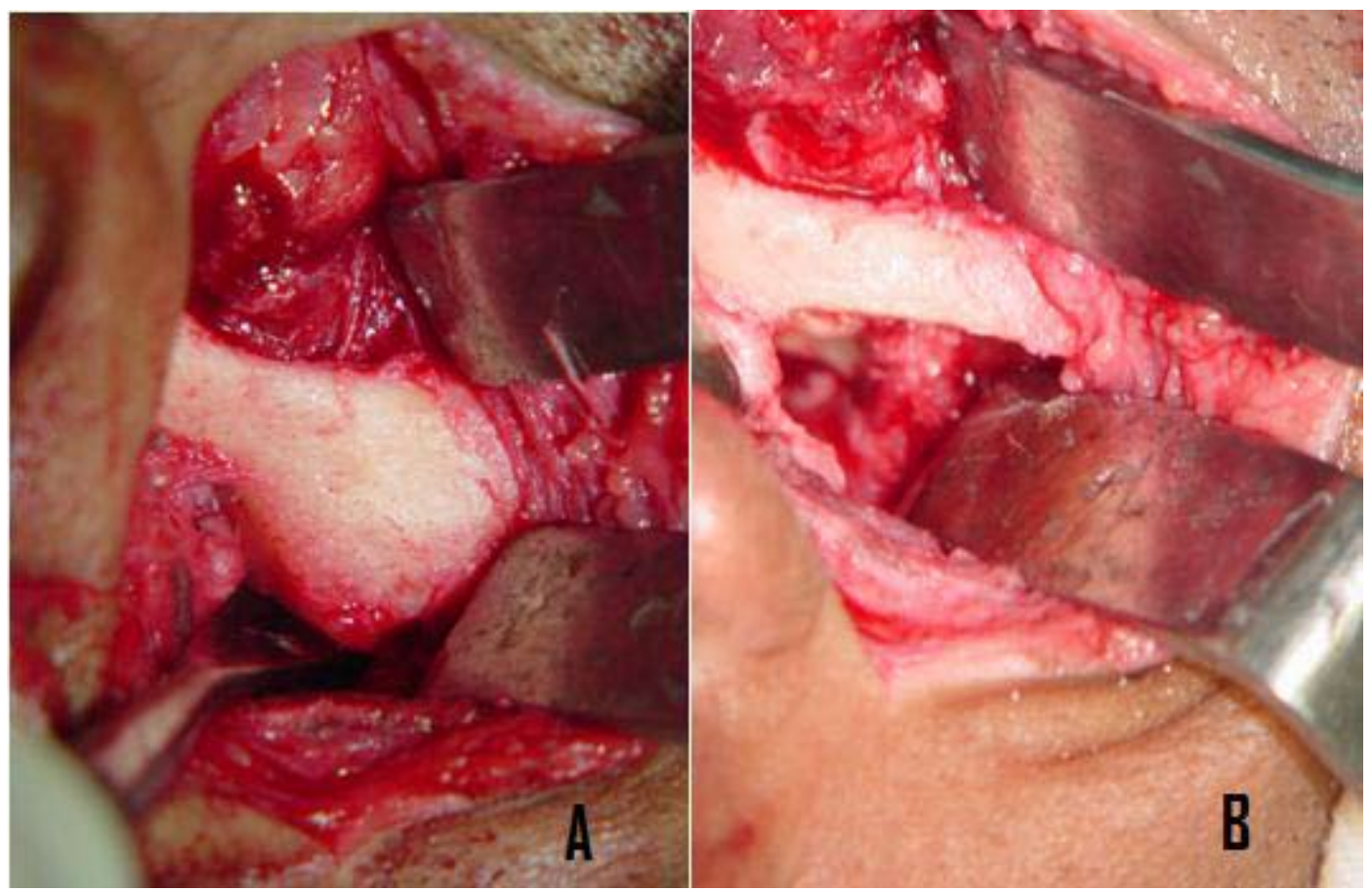

Fig. 1. a) Articular eminence; b) The removal of the articular eminence (eminectomy). 
Table 1. Data of the patients treated by eminectomy according to age, sex, maximal mouth opening (MMO), presence of pre- and postoperative pain and sounds, follow-up and frequency of luxations.

\begin{tabular}{|c|c|c|c|c|c|c|c|c|c|c|}
\hline & & & \multicolumn{1}{|c|}{$\boldsymbol{M M O}-\boldsymbol{m m}$} & \multicolumn{2}{|c|}{$\boldsymbol{P A I N}$} & \multicolumn{2}{|c|}{ SOUND } & \multicolumn{2}{l|}{} \\
\hline N & Sex & Age & Pre op. & Post & Pre op & Post & Pre op & Post & Follow $^{1}$ & Luxa. $^{2}$ \\
\hline 1 & M & 24 & 53 & 46 & No & No & No & No & 63 & 2 \\
\hline 2 & F & 24 & 52 & 47 & Yes & No & No & No & 62 & 40 \\
\hline 3 & M & 40 & 47 & 45 & No & No & No & Yes & 62 & 3 \\
\hline 4 & M & 27 & 55 & 45 & No & No & Yes & No & 61 & 3 \\
\hline 5 & F & 24 & 51 & 44 & No & Yes & No & No & 53 & 28 \\
\hline 6 & F & 22 & 50 & 41 & Yes & No & Yes & Yes & 38 & 14 \\
\hline 7 & F & 52 & 61 & 32 & No & No & Yes & No & 17 & 21 \\
\hline 8 & M & 29 & 30 & 36 & No & No & No & No & 5 & 21 \\
\hline 9 & F & 27 & 43 & 40 & Yes & No & No & No & 10 & 7 \\
\hline 10 & F & 45 & 42 & 37 & Yes & Yes & Yes & Yes & 3 & 1 \\
\hline
\end{tabular}

N: number; Pre op.: pre-operative; Post: post-operative; Follow: follow-up; Luxa.: luxation; M: male; F:

female.

1. Follow-up in months.

2. Luxation frequency in months.

(range, 22-52 years). The mean duration of postoperative follow-up was 37.4 months (range, 2-63 months). There was a total of 20 eminectomies for the treatment of chronic mandibular dislocation in all 10 patients.

The mean pre- and postoperative MMOs were $48.4 \pm 8.5$ $\mathrm{mm}$ and $41.3 \pm 5.0 \mathrm{~mm}$, respectively.

There were sounds in the TMJ in four patients preoperatively, and two continued with this sign after surgery. In one patient with no preoperative sounds, crepitation was observed postoperatively.

Out of four patients that reported pain preoperatively, only one continued with this symptom. One patient, who did not report pain preoperatively, felt some pain in the postoperative period.

No nerve paralysis or recurrence of mandibular dislocation was observed in any patient.

\section{Discussion}

Surgery is often appropriate when dislocation is prolonged or recurrent (9). Numerous surgical procedures for habitual dislocation have been described in the literature based on creating a mechanical obstacle in the condylar path, such as positioning the disk anterior to the condyle, downfracturing of the zygomatic arch and fixation medial to the eminence or by the insertion of implants into the eminence $(6,9)$. In addition, there are other modes of treatment that aim to restrict movement of the condyle such as the injection of sclerosing substances or even the induction of fibrosis in the tissues adjacent to the joint $(6,10)$. Another type of treatment is to remove the mechanical obstacles in the condylar path; one such procedure is eminectomy, which was in- troduced by Myrhaug in $1951(1,7,10)$ and has been used with satisfactory results and efficacy according to the literature (11-16).

Each form of treatment has its own advantages and disadvantages. The placement of a titanium miniplate in the articular eminence aims to prevent hyperexcursion of the condyle, thereby avoiding its displacement. It has the advantage of being a reversible and less invasive method, but has the disadvantage of leading to a decrease in MMO $(6,10)$. In addition to these disadvantages, there is also the possibility of the plate fracturing, requiring a further operation for removal of the device and choice of a new treatment.

The placement of an obstacle in the articular eminence may result in some degree of limitation of mouth opening. Removing the eminence may lead to hypermobility, which may cause a degeneration of the joint and an excessive mouth opening (9). This being so, it was to be expected that the mean preoperative MMO would be lower than the postoperative one. Nevertheless, the opposite was observed, which might be accounted for by the presence of fibrosis, due to the surgical procedure itself. In addiction, the eminectomy associated with temporal fascia flap previously may determinate a steep (7).

The removal of the articular eminence, even when performed with rotary instruments may leave rugged areas that could lead to crepitation of the bone during the movement of mouth opening. This is the likely explanation for the presence of crepitation in the TMJ only postoperatively patient number 3 .

The major complaint of patients is the condyle lock- 
ing anterior to the eminence itself, because it leads to a stretching of articular components, causing pain. As a result, there is a complete remission of the symptomatology after treatment. No causal relationship was found for the pain reported by patient number 5 and the pain that failed to subside in patient number 10 was likely due to the patient's short postoperative follow-up. Regardless of the surgical approach used to gain access to the TMJ, the final dissection places the facial nerve at risk for damage (17). A loss of function of the frontalis and orbicularis oculi muscles is always a possibility (18). The incidence of complications, such as injury of the facial nerve, is very low (18), with rates varying from 9 to $18 \%$ (19) and 1.5 to $32 \%(17)$, and any resulting lesion usually disappears within 6 months. Thus the right choice of technique for making the approach to the TMJ in this study was seen to have been made, since no facial nerve paralysis was observed in any of the cases. None of the patients had hemorrhage in the transoperative procedure.

Eminectomy was shown to be efficient in the treatment of chronic mandibular dislocations in relation to postoperative MMO, recurrence and articular function.

\section{References}

1. Cardoso AB, Vasconcelos BC, Oliveira DM. Comparative study of eminectomy and use of bone miniplate in the articular eminence for the treatment of recurrent temporomandibular joint dislocation. Braz J Otorhinolaryngol. 2005;71:32-7.

2. Hale RH. Treatment of recurrent dislocation of the mandible: review of literature and report of cases. J Oral Surg. 1972;30:527-30.

3. Daelen B, Thorwirth V, Koch A. Treatment of recurrent dislocation of the temporomandibular joint with type A botulinum toxin. Int J Oral Maxillofac Surg. 1997;26:458-60.

4. Laskin DM. Myotomy for the management of recurrent and protracted mandibular dislocations. Trans Int Conf Oral Surg. 1973;4:264-8.

5. Gould JF. Shortening of the temporalis tendon for hypermobility of the temporomandibular joint. J Oral Surg. 1978;36:781-3.

6. Puelacher WC, Waldhart E. Miniplate eminoplasty: a new surgical treatment for TMJ-dislocation. J Craniomaxillofac Surg. 1993;21:176-8.

7. Myrhaug H. A new method of operation for habitual dislocation of the mandible; review of former methods of treatment. Acta Odontol Scand. 1951;9:247-60.

8. House JW, Brackmann DE. Facial nerve grading system. Otolaryngol Head Neck Surg. 1985;93:146-7.

9. Gay-Escoda C. Eminectomy associated with redirectioning of the temporal muscle for treatment of recurrent TMJ dislocation. J Craniomaxillofac Surg. 1987;15:355-8.

10. Sato J, Segami N, Nishimura M, Suzuki T, Kaneyama K, Fujimura K. Clinical evaluation of arthroscopic eminoplasty for habitual dislocation of the temporomandibular joint: comparative study with conventional open eminectomy. Oral Surg Oral Med Oral Pathol Oral Radiol Endod. 2003;95:390-5.

11. Kuttenberger JJ, Hardt N. Long-term results following miniplate eminoplasty for the treatment of recurrent dislocation and habitual luxation of the temporomandibular joint. Int J Oral Maxillofac Surg. 2003;32:474-9.

12. Pogrel MA. Articular eminectomy for recurrent dislocation. Br J Oral Maxillofac Surg. 1987;25:237-43.

13. Irby WB. Surgical correction of chronic dislocation of the tem- poromandibular joint not responsive to conservative therapy. J Oral Surg (Chic). 1957;15:307-12.

14. Oatis GW Jr, Baker DA. The bilateral eminectomy as definitive treatment. A review of 44 patients. Int J Oral Surg. 1984;13:294-8.

15. Hale RH. Treatment of recurrent dislocation of the mandible: review of literature and report of cases. J Oral Surg. 1972;30:527-30.

16. Helman J, Laufer D, Minkov B, Gutman D. Eminectomy as surgical treatment for chronic mandibular dislocations. Int J Oral Surg. 1984;13:486-9.

17. Lovely FW, Copeland RA. Reduction eminoplasty for chronic recurrent luxation of the temporomandibular joint. J Can Dent Assoc. $1981 ; 47: 179-84$.

18. Vasconcelos BC, Bessa-Nogueira RV, Cypriano RV. Treatment of temporomandibular joint ankylosis by gap arthroplasty. Med Oral Patol Oral Cir Bucal. 2006;11:E66-9.

19. Hong Y, Gu X, Feng X, Wang Y. Modified coronoid process grafts combined with sagittal split osteotomy for treatment of bilateral temporomandibular joint ankylosis. J Oral Maxillofac Surg. 2002;60:11-8. 